control cancer pain. ${ }^{1,2}$ However, we later encountered advanced morphine tolerance. ${ }^{3}$ Since intrathecal alpha-adrenergic agonists produce analgesia in animal models ${ }^{4}$ we began to study such agents (clonidine) to see if they could be used to initiate intrathecal analgesia when advanced systemic and spinal narcotic tolerance had developed in terminal cancer patients. The first step, since we knew of no prior use intrathecally in man, was to evaluate neurotoxicity in a chronic animal model. ${ }^{5}$ With this reassurance, we proceeded with trials of bolus intrathecal clonidine in cancer patients, ${ }^{6}$ followed by chronic intrathecal infusion in patients with advanced morphine tolerance. ${ }^{7}$ A similar approach was taken by Yaksh et al, using the intrathecal delta agonist D-ala ${ }^{2}-\mathrm{D}-\mathrm{leu}^{5}$-enkephalin (DADLE). ${ }^{8}$ The knowledge gained from these studies on neurotoxicity and efficacy justified further careful study of both $\alpha_{2}$-agonists (such as clonidine) and DADLE in the setting of cancer pain.

Since the earliest reports of successful intraspinal narcotic analgesia, a host of agents have been tried intraspinally, often in non-cancer patients. Most of this work has been done without studies to check on the absence of spinal cord neurotoxicity. Clonidine and related $\alpha_{2}$-agonists theoretically have the potential to affect adversely regional spinal cord blood flow, systemic vascular resistance, and heart rate. ${ }^{6,9}$ It might have been practically acceptable, though ethically debatable, for clonidine to have been tried in terminally ill patients before studies of neurotoxicity had been complete but our desire to help in difficult pain cases, such as those reported by Tamsen and Gordh, should not lead us to sacrifice a measured, scientific approach to the study of new intraspinal agents. Such agents must be free of serious neurotoxicity and deliverable in the long term without serious side-effects, addiction, or tolerance. These limitations are illustrated in a detailed report of observations during chronic intrathecal clonidine use in a cancer pain patient. ${ }^{10}$

Department of Surgery,

Dartmouth-Hitchcock Medical Center

Dartmouth, New Hampshure, USA

DENNIS W. COOMBS

1. Coombs DW, Saunders R, Gaylor M, Pageau M, Letth M, Schatberger C. Continuous epidural narcotic analgesia via an implanted infusion device Lancet 1981; 11 * 425-26.

2. Coombs DW, Saunders RL, Block A, Gaylor MS, Colton T, Pageau ME. Continuous chronic pain relief by intraspinal narcotics infused via an implanted reservorr. fAMA 1983; 250: 2336-39.

3. Coombs DW, Maurer LH, Saunders RL, Gaylor M. Outcomes and complications of continuous intraspinal narcotic analgesia for cancer pain control. $7 \mathrm{Clm} \mathrm{Oncol}$ (in press)

4. Yaksh TL. Spinal opiate analgesia: Characteristıcs and principles of action. Pain 1981; $11 \cdot 293-346$

5. Coombs DW, Allen C, Meier FA, Fratkın JD. Chronic intraspinal clonıdine in sheep. Regi Anesth 1984; 9: 47-48.

6. Coombs DW, Saunders R, Gaylor M, LaChance D, Jensen L. Clinical trial of intrathecal clonidine for cancer pain. Regi Anesth 1984; 9: 34-35

7 Coombs DW, Savage S, LaChance D, Saunders R, Jensen L, Gaylor $M$ Intrathecal clonidine control of cancer pain when spinal morphine tolerance develops Pain 1984; (suppl 2): 21.

8. Onofrio BM, Yaksh TL. Intrathecal delta-receptor ligand produces analgesia in man. Lancet 1983; 1: 1386-87.

9. K10wsk1 W, Hulthen UL, Ritz R, Buhler FR. Alpha, adrenocepror-mediated vasoconstriction of arteries. Clm Pharmacol Ther 1983; 34: 565-69.

10. Coombs DW, Saunders RL, LaChance D, Savage S, Ragnarsson TS, Jensen LE. Intrathecal morphine tolerance: Use of intrathecal clonidine, DADLE, and intraventricular morphine. Anesthesiology (in press).

\section{SCREENING AND BREAST CANCER}

SIR,-We should like to expand on our previous reply (Sept 8 , p 574) to Professor Baum and Dr MacRae (Aug 25, p 462), who have raised some important issues about our two case-control studies ${ }^{1,2}$ on the effect on breast cancer mortality of early detection programmes. Baum and MacRae state that the results "can only be interpreted to mean that patients who have short diagnosis-to-death tımes are unlikely to have had their breast cancer diagnosed by screening. Screened patients will be likely to have longer diagnosisto-death intervals, but this does not necessarily mean that death is delayed too-ie, in addition to diagnosis being made earlier". This conclusion is incorrect, since the two studies do demonstrate a delay in the time of death, with the caveats normal to an observational case-control study. The flaw in their reasoning seems to stem from a misconception about how the two case-control studies were designed. These studies were designed to compare the breast cancer mortality rates in two groups of women, both free of disease (ie, not diagnosed with breast cancer) before entry to the study-one group who agreed to attend the screening programme and one group who refused.

As in a randomised trial or in a prospective study, all deaths from breast cancer in the first seven years after the start of the study were recorded. These deaths were in women with breast cancer diagnosed after the start of the study. In a randomised trial, these deaths would be related to the total women-years of follow-up in the two groups, to obtain the absolute mortality rates from breast cancer in the screened and unscreened groups. In our studies, absolute mortality rates were not estimated since this would have required abstracting the entire population lists of the two cities for each year of the study, to identify completely the group of refusers. The ratio of the two mortality rates, however, can be estimated very much more economically by sampling the population lists, from which sample the proportions of the relevant female population who attend or refuse screening can be determined. This sampling scheme forms the basis of the case-control approach. In the first seven years after screening started, the breast cancer mortality rate in the women accepting screening was only $30 \%$ (Utrecht) and $50 \%$ (Nijmegen) of the breast cancer mortality rate in the groups who refused screening. Since at the start of the study, both groups were free of breast cancer, advancing the time of diagnosis of breast cancer will not in itself lead to a subsequent reduction in the mortality rate unless the date of death is delayed.

The only difference in principle between these two case-control studies and a randomised trial such as the New York Health Insurance Plan (HIP) study is the process of randomisation. Both approaches aim at comparing the reduction in breast cancer mortality in a screened and an unscreened group, both of which were initially free of disease; questions of lead time as an explanation of an observed reduction are irrelevant to both designs.

The problem with the case-control approach is precisely the lack of randomisation, and the possible biases present when groups are self-selected. This question was discussed at some length ${ }^{1,2}$ and no evidence for appreciable bias was apparent. Furthermore, the HIP results suggest that whatever bias may be present might reduce rather than inflate the estimated effect of breast cancer screening, since women who refused screening were at lower risk.

The curious feature of the results of the two case-control studies referred to by Baum and MacRae in their penultimate paragraphthat the proportion accepting screening in the control group differs substantially from the proportion accepting screening overall-is easily explained. The screening history of the controls was taken only up to the time of diagnosis of the matched case. If the full screening history is considered, then the control group is similar to the total population.

Baum and MacRae are right to urge caution in the interpretation of case-control studies, since without randomisation one cannot be sure that bias has been eliminated. On this occasion, however, their criticism is aimed at the wrong target. The studies under discussion do address the fundamental question-Does screening delay death? Unless severe bias were operating (a separate issue) the results of these two studies do indicate that breast cancer screening delays death from breast cancer.

Departments of Social Medicine,

Radiology, and Pathology,

University of Nilmegen, Netherlands; Department of Pathology,

Canısius-Wilhelmına Hospital, Nijmegen;

and Department of Epıdemiology,

Unversity of Limburg

A. L. M. Verbeek

J. H. C. L. HendRiks

R. HOLLAND

M. MravunaC

F. STURMANS

Unit of Biostatistics and Field Studies,

Division of Epidemiology and Biostatistics,

International Agency for Research on Cancer, 69372 Lyon, France

N. E. DAY

Preventicon,

Utrecht, Netherlands

H. J. A. Collette

J. J. ROMBACH

F. DE WAARD

1. Verbeek ALM, Hendriks JHCL, Holland R, Mravunac M, Sturmans F, Day NE Reduction of breast cancer mortality through mass screening with modern mammography: First results of the N11megen Project, 1975-1981 Lancet 1984; mammog $1222-24$

2. Collette HJA, Day NE, Rombach JJ, de Waard F Evaluation of screenng for breast cancer in a non-randomised study (the DOM project) by means of a case-control study. Lancet 1984; 1 1224-26 